\title{
PPAR Disruption: Cellular Mechanisms and Physiological Consequences
}

\author{
Nicolas Rotman, Zofia Haftek-Terreau, Sandra Lücke, Jérôme Feige, Laurent Gelman, Béatrice \\ Desvergne, and Walter Wahli*
}

\begin{abstract}
Endocrine disruption is defined as the perturbation of the endocrine system, which includes disruption of nuclear hormone receptor signalling. Peroxisome proliferator-activated receptors (PPARs) represent a family of nuclear receptors that has not yet been carefully studied with regards to endocrine disruption, despite the fact that PPARs are known to be important targets for xenobiotics. Here we report a first comprehensive approach aimed at defining the mechanistic basis of PPAR disruption focusing on one chemical, the plasticizer monethylhexyl phthalate (MEHP), but using a variety of methodologies and models. We used mammalian cells and a combination of biochemical and live cell imaging techniques to show that MEHP binds to PPAR $\gamma$ and selectively regulates interactions with coregulators. Micro-array experiments further showed that this selectivity is translated at the physiological level during adipocyte differentiation. In that context, MEHP functions as a selective PPAR modulator regulating only a subset of PPAR $\gamma$ target genes compared to the action of a full agonist. We also explored the action of MEHP on PPARs in an aquatic species, Xenopus laevis, as many xenobiotics are found in aquatic ecosystems. In adult males, micro-array data indicated that MEHP influences liver physiology, possibly through a cross-talk between PPARs and estrogen receptors (ER). In early Xenopus laevis embryos, we showed that PPAR $\beta / \delta$ exogenous activation by an agonist or by MEHP affects development. Taken together our results widen the concept of endocrine disruption by pinpointing PPARs as key factors in that process.
\end{abstract}

Keywords: Adipogenesis · Embryonic development · Endocrine disruption · Phthalates · PPAR

\section{Introduction}

Endocrine disruption (ED) is now commonly accepted as the perturbation of the endocrine system via effects on nuclear hormone receptor signalling, or on hormone production or clearance. Originally restricted to steroid signalling, the concept has thus evolved to extend to other mem-

${ }^{\star}$ Correspondence: Prof. W. Wahl Center for Integrative Genomics

National Research Center Frontiers in Genetics

University of Lausanne

Le Génopode

$\mathrm{CH}-1015$ Lausanne

Tel.: +4121692 4110

Fax: +4121692 4115

E-mail : walter.wahli@unil.ch bers of the nuclear receptor family.[1] This family represents one of the major classes of transcription regulators in metazoans, with 48 members in human regulating functions as diverse as reproduction, differentiation, development, metabolism, and homeostasis. Within this family, peroxisome proliferator-activated receptors (PPARs) form a subgroup of three isotypes, PPAR $\alpha$ (or NR1C1), PPAR $\beta / \delta$ (or NR1C2, also known as FAAR or NUC1) and PPAR $\gamma$ (or NR1C3), which are activated by a broad range of fatty acids and their derivatives. PPARs control many vital processes, such as glucose and lipid metabolism and inflammation, as well as a variety of developmental programs..$^{[2-4]}$ They were independently identified in the early 1990 s in mice ${ }^{[5]}$ and Xenopus laevis, ${ }^{[6]}$ and were subsequently also found in fish, chicken, hamster, rat and human (for a review ${ }^{[2]}$ ). Several factors make PPARs important targets for endocrine disruption. First, increasing evidence demonstrates that PPARs and estrogen receptor pathways (the original paradigm for endocrine disruption) are cross-regulated, or at least are involved in cross-talks. ${ }^{[7-9]}$ Second, PPARs are activated by numerous xenobiotics. Third, cytochrome $\mathrm{P} 450$ genes that have been used for decades as biomark- ers for drug treatments are targets of PPARs in the liver. ${ }^{[10]}$ Indeed, these receptors were named because of their involvement in the mediation of a pleiotropic response to chemicals that provoke peroxisome proliferation in rodents. Monethylhexyl phthalate (MEHP), a primary metabolite of diethylhexyl phthalate (DEHP) is one of those peroxisome proliferators $^{[11]}$ and has been shown to activate PPARs in cell culture systems. ${ }^{[12,13]}$ Phthalates are synthetic chemicals that represent ubiquitous environmental pollutants because they are intensively used by the chemical industry and are thus produced in a very high volume (more than 8 million tonnes are used each year). Indeed phthalates are used in perfumes, paints, industrial plastics and some medical devices. However, they primarily serve as plasticizers used to soften polyvinylchloride (PVC). Human exposure to phthalates is significant, in particular exposure to DEHP, the most abundant phthalate, which is estimated to be in the range of 3 to $30 \mu \mathrm{g} / \mathrm{kg}$ body mass in the general population. ${ }^{[14]}$

Focusing on DEHP, and more precisely on one of its metabolites, MEHP, the present study aimed at characterizing the molecular mechanisms and the physiologi- 
cal consequences of disruption of PPAR pathways by endocrine disruptors. Our approach was based on two different and complementary models: mammalian cells on one hand and Xenopus laevis embryos and adults on the other hand. We used mammalian cells first to dissect the molecular action of MEHP on PPARs, with a particular emphasis on PPAR $\gamma$, and second to characterize the physiological outcomes of PPAR disruption using the paradigm of adipogenesis. This approach should reflect human exposure to DEPH/MEHP, and we defined the doses of our treatments accordingly. As already mentioned, PPAR activation is tightly linked to the metabolism of xenobiotics in the liver. We were thus interested in studying the consequences of the disruption of PPARs in that organ and we chose an aquatic animal, the amphibian Xenopus laevis, for that purpose. Since ED has been extensively studied for its impact on embryonic development we addressed PPAR endocrine disruption using Xenopus laevis embryos.

Today, a clear mechanistic understanding is lacking, which would help in establishing appropriate environmental regulations, but would also provide a key fundamental basis to the concept of endocrine disruption. Our work should pave the way towards a clearer understanding as we characterize MEHP as a selective PPAR modulator (SPPARM) and show some physiological consequences of PPAR disruption by this substance.

\section{Results}

\section{MEHP Can Interfere with PPAR Signalling}

In transient transactivation experiments, ${ }^{[12,13]}$ previous studies have reported that MEHP could activate mouse and human PPARs. We wanted to reproduce those results in mice and to extend them to Xenopus PPARs. Appropriate cells were transfected with a PPRE-firefly luciferase reporter construct and an expression vector coding for mouse $\operatorname{PPAR} \alpha, \operatorname{PPAR} \beta / \delta, \operatorname{PPAR} \gamma$ or their three $X e$ nopus laevis orthologs. After transfection, cells were treated with 1-200 $\mu \mathrm{M}$ MEHP or with the following selective agonists: Wy 14,643 (mPPAR $\alpha, 10 \mu \mathrm{M}), \mathrm{L} 165041$ (5 $\mu \mathrm{M}$ for $\mathrm{mPPAR} \beta / \delta$ and $20 \mu \mathrm{M}$ for $\mathrm{xPPAR} \beta / \delta$ ), rosiglitazone (mPPAR $\gamma, 1 \mu \mathrm{M})$, ETYA (5,8,11,14-eicosatetraynoic acid, PPAR $\alpha$, $10 \mu \mathrm{M}$ ), and BRL49653 (xPPAR $\gamma, 5 \mu \mathrm{M})$. Except for xPPAR $\alpha$ all the different PPAR isotypes could be activated by MEHP. However, high concentrations of MEHP were necessary to reach the level observed when the different selective agonists were used (32 $\mu \mathrm{M}$ for mPPAR $\alpha, 50 \mu \mathrm{M}$ for xPPAR $\gamma$ and $200 \mu \mathrm{M}$ for all the other PRARs tested).

We also wanted to evaluate more precisely the species specificity of PPAR acti- vation by MEHP. It appears that mPPAR $\alpha$ is very sensitive to MEHP, whereas XPPAR $\alpha$ is not (at least at $200 \mu \mathrm{M}$ ). This should be compared with the fact that Wy 14,643, which is a very potent agonist of mPPAR $\alpha$ can barely activate xPPAR $\alpha$. On the contrary, $\mathrm{xPPAR} \beta / \delta$ and $\mathrm{xPPAR} \gamma$ appear to be more sensitive to MEHP than their mouse orthologs.

In summary, mouse and Xenopus PPARs are sensitive to MEHP with species-specific differences in their potencies.

\section{Molecular Characterization of PPAR $\gamma$ Disruption by MEHP}

To understand the mechanism by which MEHP could alter PPAR signalling, we asked step-wise questions.

The first one was to evaluate the binding mode of MEHP in the PPAR $\gamma$ ligand binding pocket (LBD) through modeling, and then compare it to the binding of rosiglitazone, available from the crystal structure of the PPAR $\gamma$ LBD in complex with this agonist. Docking of MEHP in hPPAR $\gamma$ showed that both the $R$ - and the $S$-enantiomer of MEHP could fit in the PPAR $\gamma$ LBD, very similar to the way rosiglitazone does. In fact, MEHP and rosiglitazone bind to the PPAR $\gamma$ LBD in similar conformations where only one side of the T-shaped binding pocket is occupied and where similar residues are contacted. In contrast, DEHP could not fit into it as its carboxylate function is esterified by a bulky and hydrophobic chain. ${ }^{[15,16]}$

Since MEHP is capable of acting as an agonist bound to the ligand binding domain of PPAR $\gamma$, the next step was to evaluate whether this binding occurs in living cells and results in coregulator recruitment. Coregulator recruitment is indeed the major functional determinant, since it follows conformational changes of the nuclear receptor LBD in response to ligand binding, and assists PPAR $\gamma$ to regulate transcription. For that purpose, we used fluorescence correlation spectroscopy (FCS), which is a technique measuring the diffusion of fluorescent molecules at high temporal resolution that can be used in the living cell. We first demonstrated that the fusion protein YFP-PPAR exhibits reduced mobility upon ligand binding ${ }^{[17]}$ due to the formation of large complexes through coactivator recruitment. ${ }^{[18,19]}$ Exposing the cells to MEHP also reduced YFP-PPAR $\gamma$ mobility, although to a lesser degree than rosiglitazone, suggesting that the size of PPAR $\gamma /$ coactivator complexes formed by MEHP binding is smaller and thus different from those formed in response to rosiglitazone. [15]

To identify the regulators recruited by rosiglitazone and MEHP in the native context of a living cell, we first established the appropriate tools for evaluating PPAR activity and protein-protein interaction via fluorescence resonance energy transfer
(FRET) imaging. ${ }^{[17,20]}$ We were then able to demonstrate that MEHP only partially abolished the strong interaction between PPAR $\gamma$ and the corepressor NCoR observed in the absence of a ligand. With respect to coactivators, MEHP enhanced the recruitment of the Med1 subunit of the mediator complex, just as rosiglitazone did. However, it was totally inefficient in inducing the recruitment of p300, another coactivator recruited by PPAR $\gamma$ in the presence of rosiglitazone. In contrast, and as determined by in vitro assays, the interaction of PPAR $\gamma$ with PPAR $\gamma$ coactivator $1-\alpha$ (PGC- $1 \alpha$ ) was strongly enhanced by MEHP but only modestly enhanced by rosiglitazone. ${ }^{[15]}$

We finally needed to assess whether this selective recruitment also operates when PPAR $\gamma$ is bound to DNA on target promoters. Using a specific antibody against each of the coregulators, we performed chromatin immuno-precipitation (ChIP) and assessed PPAR $\gamma$ target promoter occupancy, in the presence of either rosiglitazone or MEHP. Consistent with our FRET and pulldown observations, MEHP induced a partial clearance of NCoR from the two GyK and OLR1 PPAR $\gamma$ target promoters. MEHP did not promote the recruitment of p300 or SRC-1 on target promoters. However, it did induce a recruitment of Med1 to slightly lower levels than those achieved by rosiglitazone, but promoted PGC- $1 \alpha$ recruitment at much higher levels. ${ }^{[15]}$

In conclusion, these mechanistic studies showed that the endocrine disruptor MEHP binds to PPAR $\gamma$ and selectively regulates interactions with coregulators in living cells (Fig. 1). This selectivity strikingly correlates with the global pattern of promoterspecific and ligand-dependant coregulator recruitment.[21]

\section{PPAR $\gamma$ Disruption and the Adipogenic Program}

To investigate the interference of MEHP with PPAR $\gamma$ regulated pathways at the cellular level, we studied the influence of MEHP on adipogenesis, a well-characterized PPAR $\gamma$ regulated function. For that purpose we compared the ability of MEHP and of rosiglitazone to promote 3T3L1 preadipocyte differentiation in mature adipocytes. The maximal effect of MEHP was obtained at $100 \mu \mathrm{M}$ but remained lower than that induced by rosiglitazone. The actions of both ligands on adipocyte differentiation were confirmed by quantification of cellular triglyceride contents, which were strongly induced by rosiglitazone but only partially induced by MEHP. Thus, although MEHP significantly induces adipocyte differentiation, this phthalate monoester has a reduced adipogenic efficacy compared to that of rosiglitazone, most likely due to the selective modulation of its activity through 


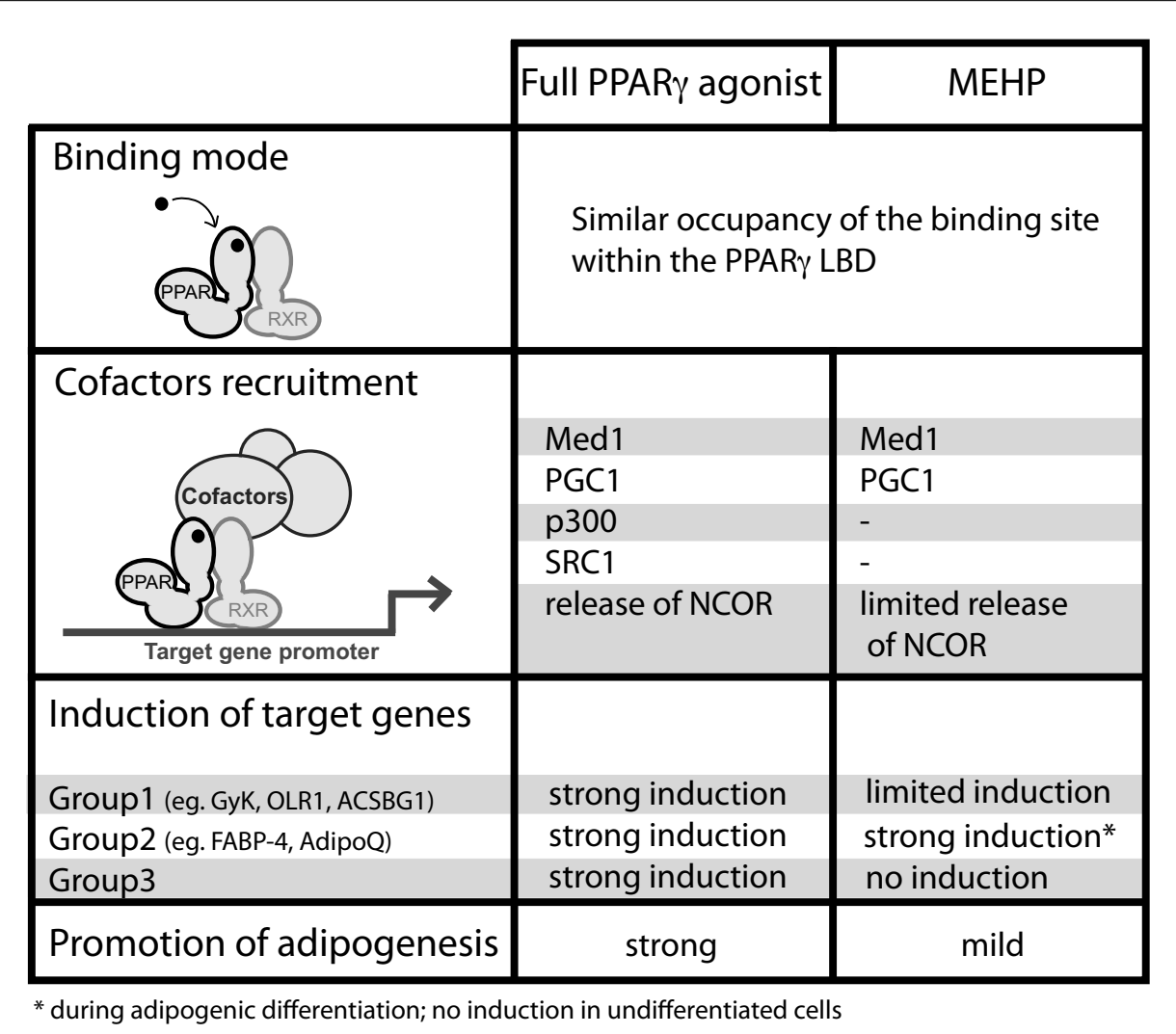

Fig. 1. MEHP is a selective PPAR $\gamma$ modifier in mammalian cells. Whereas monethylhexyl phthalate (MEHP) and rosiglitazone (full PPAR $\gamma$ agonist) have similar binding modes within the PPAR $\gamma$ pocket, they induce the recruitment of different cofactors on target gene promoters. This difference is translated into differential gene expression and, finally, differential adipogenic potency. ACSBG1, acyl-CoA synthetase Bubblegum 1; AdipoQ, adiponectin; FABP-4, fatty acid-binding protein 4; GyK, Glycerol kinase; OLR1, oxidized low density lipoprotein receptor 1.

differential coregulator recruitment. These actions of MEHP on adipogenesis are truly linked to PPAR $\gamma$ activation, since PPAR $\gamma$ inhibition, either via expression of antiPPAR $\gamma$ siRNA or by the use of the PPAR $\gamma$ antagonist GW9662, inhibited the adipogenic actions of MEHP. ${ }^{[15]}$

As we demonstrated selective cofactor recruitment by PPAR $\gamma$ in the presence of MEHP, we hypothesized that this should also translate into a selective array of target genes. We thus pursued our exploration of the adipogenic actions of MEHP and rosiglitazone and their underlying molecular pathways, by performing gene expression array analyses on glass slides spotted at custom with a collection of 17,000 mouse cDNAs. The vast majority of the genes regulated by MEHP were also regulated by rosiglitazone, again indicating that the adipogenic actions of MEHP are mediated by PPAR $\gamma$. MEHP specifically regulated only a very low number of genes, which could not be assigned to characterized adipocyte differentiation pathways. In contrast, rosiglitazone had a broader action than MEHP since this full agonist specifically regulated around $30 \%$ of the genes. Thus, MEHP potentially acts as a selective PPAR modulator regulating only a subset of PPAR $\gamma$ target genes compared to the action of a full agonist ${ }^{[15]}$ (Fig. $1)$.

A limitation to the interpretation of these data is that it is difficult to uncouple the direct induction of PPAR $\gamma$ target genes from their indirect activation via the global network of transcription factors controlling the adipogenic program. Thus, the same gene expression analyses were repeated with 3T3L1 cells treated for $48 \mathrm{~h}$ with rosiglitazone or MEHP, either before differentiation or after differentiation with a classic adipogenic cocktail. Quantitative expression analyses of a subset of genes demonstrated that, in the same cellular context, MEHP exerts a selective action on different PPAR $\gamma$ target genes and that this varies according to the differentiation status of the cell. Two different classes of genes must be distinguished. The first group includes genes such as GyK, OLR1 and ACSBG1 on which MEHP exerts a lower activity than rosiglitazone, independently of the differentiation status of the cell. A second group is exemplified by FABP-4 and AdipoQ, which similarly respond to MEHP and rosiglitazone during differentiation but are principally induced by rosiglitazone in un- differentiated cells. ${ }^{[15]}$ These two categories probably reflect the interplay between the ligand-specific cofactor complex formed by PPAR $\gamma$ and the promoter structure and sequence to which it binds.

Our results on the adipogenic action of MEHP strongly argue that in addition to an action on hepatic carcinogenesis through PPAR $\alpha$-mediated peroxisome proliferation, ${ }^{[22]}$ the endocrine-disrupting actions of DEHP through its MEHP metabolite should also be considered with respect to the development of metabolic disorders. On a more fundamental note, our results also point to the importance of the cellular context regarding coregulator abundance, illustrated by the differences of PPAR $\gamma$ transactivation by MEHP according to the cell line investigated. This suggests that PPAR $\gamma$ agonists may exert different effects on subpopulations of adipocytes and their progenitors in the adipose tissue, an aspect that should be taken into consideration in pharmacological strategies aimed at finding PPAR $\gamma$ modulators that act on adipocyte physiology.

\section{PPAR Disruption in Xenopus laevis Liver}

Having shown that MEHP could activate Xenopus laevis PPARs (with the exception of PPAR $\alpha$ ), we were particularly interested in identifying the pathways that this chemical could alter in the liver, the major detoxifying organ, of that aquatic species. Because PPAR metabolism in Xenopus liver had not been studied previously, it was essential to identify the genes activated or repressed upon PPAR activation by a characterized agonist. Given that XPPAR $\alpha$ and $\mathrm{xPPAR} \beta / \delta$ are very abundant in the male adult liver, whereas XPPAR $\gamma$ is only weakly expressed, we chose to use ETYA, which activates both $\mathrm{xPPAR} \alpha$ and $\mathrm{xPPAR} \beta / \delta{ }^{[23]}$ Finally, we were also interested in characterizing the pathways induced or repressed upon estrogen (17 $\beta$-estradiol, E2) treatment reasoning that a comparison between the ETYA, E2 and MEHP treatments should help in understanding the molecular action of MEHP on liver metabolism: would it be primarily mediated through PPARs or through a cross-talk with ERs?

Xenopus laevis adult males were treated by injecting them with a solution containing either ETYA, or MEHP, or the vehicle only, directly in one dorsal lymph bag. After $48 \mathrm{~h}$, livers were dissected, their RNA extracted and a gene expression array analysis conducted. A total of 2634 genes were regulated by the treatments (with a $\mathrm{P}$ value $\leq 0,008$, on a total of 12977 probes). The most potent treatment was, as expected, the E2 treatment, which modulated 2526 genes including known target genes such as vitellogenin or serpin. ETYA impacted on 285 genes. 75 genes were induced or repressed by MEHP, with only eleven being solely modulated by 
the phthalate (Fig. 2). This last point suggests that MEHP affects liver metabolism primarily through disruption of PPAR and/ or ER signalling. More precisely, 18 genes were modulated by the three treatments, 40 genes were coregulated by E2 and MEHP, and six genes by MEHP and ETYA (Fig. 2). It thus appears that MEHP treatment is closer to E2 treatment than to ETYA treatment. This implies that either MEHP can activate ERs independently of PPAR, or that PPAR activation by MEHP finally induces a different set of target genes than a bona fide PPAR agonist. This last hypothesis, which would represent a variation of the concept of SPPARM is tempting, given that MEHP has not been shown to directly interact with ER and should therefore be tested further.

The detailed analysis of the data to precisely address the pathways modified by the different treatments is still in progress. However, because the Xenopus laevis genome has not yet been fully sequenced, only 1071 genes have yet been successfully annotated, which complicates the task. Already at this stage, our data show that MEHP can influence liver transcriptome in Xenopus laevis, and further suggest than MEHP action is essentially mediated by PPARs and ERs.

\section{PPAR Disruption in Early Xenopus laevis Development}

The ED theory predicts that embryos are particularly sensitive to disruption by chemicals present in their environment. We wanted to evaluate this point regarding PPAR disruption by MEHP. We used
Xenopus laevis for that purpose because it is a convenient model for embryo development and because it is an aquatic species. This work, even though of a fundamental nature should thus shed light on the sharp decline observed in some wild amphibian populations worldwide. ${ }^{[24]}$ We have reported previously that XPPAR $\alpha$ is not activated by MEHP, whereas xPPAR $\beta / \delta$ and $x P P A R ~ \gamma$ are quite sensitive. Given that PPAR $\gamma$ is not expressed during Xenopus embryo development, we therefore focused on $\mathrm{xPPAR} \beta / \delta$, which is present and abundant throughout embryogenesis. ${ }^{[6]}$ Part of the work was devoted to a better understanding of the functions of xPPAR $\beta / \delta$ in early Xenopus development and will be reported elsewhere. To address the question of endocrine disruption, we subjected batches of embryos to increasing concentrations of MEHP $(50 \mu \mathrm{M}$ to $200 \mu \mathrm{M})$ or of the selective $\mathrm{xPPAR} \beta / \delta$ agonist (L165041, $0.1 \mu \mathrm{M}$ to $100 \mu \mathrm{M}$ ). Embryos were treated from cleavage stage (soon after fertilization) to feeding tadpole stage (when organogenesis is set up and before metamorphosis) when they were fixed and examined using morphometric tools. Whereas L164041 induced a slight decline in survival rate of about $10 \%$ at concentrations starting from $25 \mu \mathrm{M}$, even the highest concentrations of MEHP did not affect survival. Only subtle defects could be detected when comparing control embryos (treated with vehicle only) with embryos treated with MEHP or L165041. Among them, eye size appeared to be affected in a significant manner. L165041 treatment reduced by $18 \%$ the area occupied by the

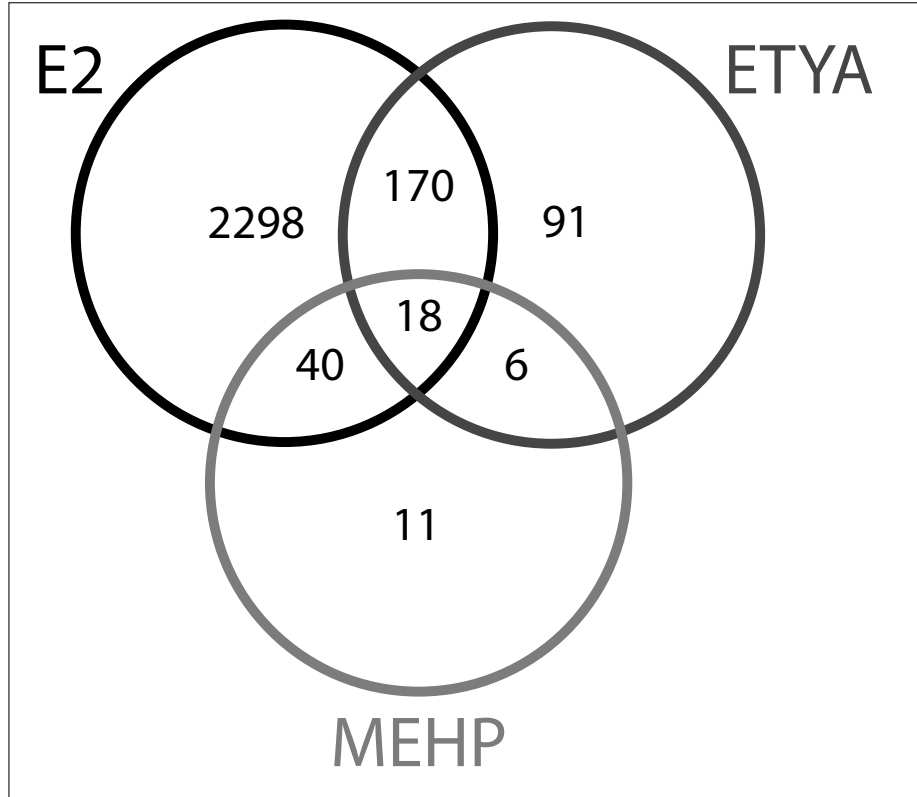

Fig. 2. MEHP primarily affects adult Xenopus laevis liver transcriptome through PPARs and ERs. Venn diagram obtained from micro-array experiments conducted on Xenopus laevis adult male livers treated with 5,8,11,14-eicosatetraynoic acid (ETYA), $17 \beta$-estradiol (E2) or monethylhexyl phthalate (MEHP).

retina $\left(20 \mu \mathrm{M}\right.$, t-test: $\left.\mathrm{p}<10^{-3}\right)$, whereas MEHP treatment increased it by $25 \%$ (200 $\mu \mathrm{M}$, t-test: $\left.\mathrm{P}<10^{-15}\right)$. This result indicated that MEHP exposure can impact on embryo development even if it did not induce dramatic effects under our experimental conditions. Additional work is necessary to evaluate whether this effect is mediated by PPAR $\beta / \delta$, but the fact that exposure to a potent selective agonist for PPAR $\beta / \delta$ also affected eye size would suggest it is. If it were true, MEHP could function in Xenopus early embryos as a selective PPAR $\beta / \delta$ modulator that would only impact on a restricted repertoire of target genes and could eventually mediate opposite physiological responses to those triggered by bona fide PPAR $\beta / \delta$ agonists: MEHP increases eye field/size, whereas L165041 decreases it. Better understanding of the PPAR $\beta / \delta$ functions in normal development will help to clarify this last idea.

\section{Discussion}

We have shown that mouse and $X e$ nopus PPARs are sensitive to MEHP. In mammalian cells we have explored further the interaction between MEHP and PPAR $\gamma$. Molecular and cellular analyses demonstrated that MEHP directly activates PPAR $\gamma$ and promotes adipogenesis, albeit to a lower extent than the full-agonist rosiglitazone. In particular, we demonstrated that MEHP induces a selective activation of different PPAR $\gamma$ target genes (Fig. 1). Chromatin immuno-precipitation and fluorescence microscopy in living cells revealed that this selective activity correlates with the recruitment of a specific subset of PPAR $\gamma$ coregulators, which includes Med1 and PGC1, but not p300 and SRC1 (Fig. 1). These results therefore highlight key mechanisms in metabolic disruption but are also instrumental in the context of selective PPAR modulation, a promising field for new therapeutic development. While these cellular observations suggest that exposure to phthalates could have detrimental effects leading to obesity, results in mouse models actually demonstrate that exposure to the phthalate plasticizer DEHP protects from diet-induced obesity (manuscript in preparation).

It is very likely that DEHP acts on PPAR $\gamma$ as a SPPARM and should therefore be capable of interfering with full agonists such as rosiglitazone.

We used the Xenopus laevis model to show that MEHP can affect the transcriptome of a whole organ, the liver, and influence early embryogenesis. Furthermore, we have shown that MEHP action on the liver was essentially mediated through PPARs and ERs with a possible cross-talk between those two sub-classes of receptors. Finally 
our work on embryogenesis indicated that even if MEHP does not affect survival it can modulate the development of such a vital organ as the eye. Both molecular and morphological observations indicated that MEHP does not function as a full PPAR agonist in Xenopus, suggesting that the concept of SPPARM might be extended from mammals to amphibians. However, further work is needed in order to describe the molecular action of MEHP in Xenopus laevis. Better understanding of the endogenous functions of PPARs in that species is a prerequisite and we are pursuing that direction.

In conclusion, our work contributes to widening the concept of ED to non-steroid nuclear hormone receptors. It also adds evidence to the already well-documented question of phthalates, and in particular MEHP, as endocrine disruptors. And finally, it validates our hypothesis that PPARs represent important targets for endocrine disruption. This last point should be taken into consideration in the process of defining whether a chemical could function as an endocrine disruptor.

\section{Experimental}

\section{Transient Transactivations Experiments}

C2C12 cells were used for mPPARs transfection, Cos-7 cells for xPPAR $\alpha$, and HeLa cells for $x P P A R \beta / \delta$ and $x P P A R ~ \gamma$. For details see [15].

\section{Analysis of Xenopus laevis Liver Metabolism}

Xenopus laevis adult male livers were exposed for $48 \mathrm{~h}$ to a single injection in dorsal lymph bags of either $16.7 \mathrm{mg} / \mathrm{kg}$ of $\mathrm{E} 2$, or $36.8 \mathrm{mg} / \mathrm{kg}$ of ETYA or $50 \mathrm{mg} / \mathrm{kg}$ of MEHP. Xenopus laevis Affymetrix ${ }^{\circledR}$ microarrays were performed in collaboration with the DNA array facility of Lausanne University.

\section{Analysis of Xenopus laevis Early Development}

Xenopus eggs were obtained by manual squeezing, fertilized in vitro by standard methods and de-jelled by treatment with $3 \%$ cysteine. Eggs were then sorted to remove imperfectly cleaved embryos and placed into $40 \mathrm{ml}$ of the different solutions for treatment, in a glass Petri dish. Embryos were left to develop until feeding tadpole stage where they were fixed in MEMFA (0.1 M MOPS pH 7.4, 2 mM EGTA, 1 $\mathrm{mM} \mathrm{MgSO}_{4}, 3.7 \%$ formaldehyde) for 1-2 $\mathrm{h}$, dehydrated and stored in $100 \% \mathrm{EtOH}$. Solutions of treatment were prepared as follows: L165041 or MEHP 1000× stock solutions were made in DMSO and diluted in $0.1 \times$ MBS $(8.8 \mathrm{mM} \mathrm{NaCl}, 0.1 \mathrm{mM}$ $\mathrm{KCl}, 0.07 \mathrm{mM} \mathrm{CaCl}_{2}, 0.1 \mathrm{mM} \mathrm{MgSO}_{4}$,
$0.5 \mathrm{mM}$ HEPES, $0.25 \mathrm{mM} \mathrm{NaHCO}{ }_{3}$, $\mathrm{pH}$ 7.8 ) to obtain final concentrations of 1,5 , $10,20,40$ and $80 \mu \mathrm{M}$ of L165041 or 50, 100 and $200 \mu \mathrm{M}$ of MEHP. DMSO final concentration was set to $1: 10000(\mathrm{v}: \mathrm{v})$ in every solution, and a control solution was made with DMSO only. Images were obtained using a stereomicroscope equipped with a digital camera. Morphometric analyzes were conducted using Photoshop CS (Adobe).

\section{Acknowledgements}

We thank the Swiss National Science Foundation (NRP50) and the Etat de Vaud for funding this research. Micro-array experiments on Xenopus laevis samples were performed in collaboration with the DNA Array Facility of Lausanne University.

Received: March 22, 2008

[1] M. M. Tabb, B. Blumberg, Mol. Endocrinol. 2006, 20, 475 .

[2] B. Desvergne, W. Wahli, Endocr. Rev. 1999, 20, 649 .

[3] S. Kersten, B. Desvergne, W. Wahli, Nature 2000, 405, 421.

[4] N. Rotman, L. Michalik, B. Desvergne, W. Wahli, in 'Advances in Developmental Biology', Ed. R. Tanejah, Elsevier, 2006, 16.

[5] I. Issemann, S. Green, Nature 1990, 347, 645.

[6] C. Dreyer, G. Krey, H. Keller, F. Givel, G. Helftenbein, W. Wahli, Cell 1992, 68 , 879.

[7] T. Suzuki, S. Hayashi, Y. Miki, Y Nakamura, T. Moriya, A. Sugswara, T. Ishida, N. Ohuchi, H. Sasano, Endocr Relat. Cancer 2006, 13, 233.

[8] C. Qin, R. Burghardt, R. Smith, M. Wormke, J. Stewart, S. Safe, Cancer Res. 2003, 63, 958 .

[9] S. B. Nunez, J. A. Medin, O. Braissant, L. Kemp, W. Wahli, K. Ozato, J. H. Segars, Mol. Cell Endocrinol. 1997, 127, 27.

[10] S. S. Lee, T. Pineau, J. Drago, E. J. Lee, J. W. Owens, D. L. Kroetz, P. M. FernandezSalguero, H. Westphal, F. J. Gonzalez, Mol. Cell Biol. 1995, 15, 3012.

[11] H. A. Dirven, P. H. van der Broek, M. C. Peeters, J. G. Peters, W. C. Mennes, B. J. Blaauboer, J. Noordhoek, F. J. Jongeneelen, Biochem. Pharmacol. 1993 , 45, 2425.

[12] C. H. Hurst, D. J. Waxman, Toxicol. Sci. 2003, 74, 297.

[13] E. K. Maloney, D. J. Waxman, Toxicol. Appl. Pharmacol. 1999, 161, 209.

[14] G. Latini, Clin. Chim. Acta 2005, 361, 20.

[15] J. N. Feige, L. Gelman, D. Rossi, V. Zoete, R. Metivier, C. Tudor, S. I. Anghel, A. Grosdidier, C. Lathion, Y. Engelborghs, O. Michielin, W. Wahli, B. Desvergne, $J$. Biol. Chem. 2007, 282, 19152.

[16] V. Zoete, A. Grosdidier, O. Michielin, Biochim. Biophys. Acta 2007, 1771, 915.

[17] J. N. Feige, L. Gelman, C. Tudor, Y. Engelborghs, W. Wahli, B. Desvergne, $J$. Biol. Chem. 2005, 280, 17880.
[18] L. Gelman, J. N. Feige, C. Tudor, Y. Engelborghs, W. Wahli, B. Desvergne, Nucl. Recept. Signal 2006, 4, e010.

[19] C. Tudor, J. N. Feige, H. Pingali, V. B. Lohray, W. Wahli, B. Desvergne, Y. Engelborghs, L. Gelman, J. Biol. Chem. 2007, 282, 4417.

[20] J. N. Feige, D. Sage, W. Wahli, B. Desvergne, L. Gelman, Microsc. Res. Tech. 2005, 68, 51.

[21] L. Gelman, J. N. Feige, B. Desvergne, Biochim. Biophys. Acta 2007, 1771, 1094.

[22] I. Rusyn, J. M. Peters, M. L. Cunningham, Crit. Rev. Toxicol. 2006, 36, 459.

[23] G. Krey, O. Braissant, F. L'Horset, E. Kalkhoven, M. Perroud, M. G: Parker, W. Wahli, Mol. Endocrinol. 1997, 11, 779.

[24] C. Carey, C. J. Bryant, Environ. Health Perspect. 1995, 103 Suppl 4, 13. 